C-12 (in XXIV), die als Partner für die Esterbindung in XIX in Frage kommt.

Durch Einfügen des Diennitril-Systems (I) in die Formel XIX ergeben sich noch 4 mögliche Formeln für Borrelidin: II, ein Isomeres IIa von II mit der Cyanogruppe an C-15 und 2 analoge Isomere mit dem Cyclopentanring als Bestandteil des grossen Lactonringes (XXXI a und $b$ ).

Verbindungen der Formeln XXXI ( $\mathrm{a}$ und b) müssten $z u$ einem Triketon (analog XI) führen, in dem die Gruppierung $-\mathrm{CO}-\mathrm{CH}_{2}-\mathrm{COOCH}_{3}$ zweimal vorhanden ist. Gemäss dem NMR-Spektrum dieser Verbindung (XI, siehe oben) liegt diese Gruppe aber sicher nur einmal vor. Formeln mit dem Ringgerüst von II muss gegenüber den Formeln XXXI a und b auch auf Grund des Massenspektrums des Methylesters III der Vorzug gegeben werden, da im Bereich höherer Massen mehrmals die Differenz 127 für den Verlust eines Carbomethoxycyclopentyl-Restes vorkommt, während die Differenz $73\left(\mathrm{CH}_{2} \mathrm{COOCH}_{3}\right)$, die bei Methylestern von XXXI zu erwarten wäre, nicht auftritt.

Zwischen den Formeln II und dem Isomeren mit CN an C-1.5 lässt sich mit Hilfe von Spin-Entkopplungs- experimenten mit dem Borrelidin-methylester (III) entscheiden: Durch Einstrahlen bei $\delta 6,2$ ppm (AlkenProton) tritt Entkopplung eines Signals bei $\delta 2,5 \mathrm{ppm}$ $\left(\mathrm{CH}_{2}\right)$ ein, während die Signale von Protonen neben Sauerstoff $(\delta 3,85 \mathrm{ppm}, 4,10 \mathrm{ppm}$ und 4,92 ppm) nicht verändert werden.

Die Mikroanalysen der kristallinen Verbindungen III, IV und XII unterstützen die angegebenen Formeln. Die übrigen, nicht kristallisierbaren und meist nicht unzersetzt destillierbaren Verbindungen wurden nicht analysiert, sondern ihre Bruttoformeln aus den Massenspektren von Acetylderivaten unter Berücksichtigung der spektroskopisch bestimmten funktionellen Gruppen berechnet.

Summary. Structure II has been determined for the antibiotic Borrelidin by chemical degradation, mass spectroscopy and NMR-spectroscopy of the degradation products.

\section{W. Keller-Schierlein}

Organisch-chemisches Laboratorium dev Eidg. Technischen Hochschule, Zürich (Schweiz), 18. März 1966.

\section{7-Hydroxy-4'-methoxy-isoflavon (Formononetin) aus Süssholzwurzel. Über Inhaltsstoffe der Süssholzwurzel. II $^{1}$}

Bei Untersuchungen über phenolische Inhaltsstoffe der Süssholzwurzel konnte aus dem methanolischen Wurzelextrakt durch Fällung mit Bleiacetat neben Liquiritin eine Fraktion erhalten werden, die mit $\mathrm{FeCl}_{3} / \mathrm{K}_{3}\left[\mathrm{Fe}(\mathrm{CN})_{6}\right]$ positive Reaktion auf Phenole gab, mit $\mathrm{Mg} / \mathrm{HCl}$ aber nicht reduziert wurde. Die Reinsubstanz $50 \mathrm{mg}$ aus $4 \mathrm{~kg}$ Wurzel) schmilzt bei $258^{\circ} \mathrm{C}$ und entspricht der Zusammensetzung $\mathrm{C}_{16} \mathrm{H}_{12} \mathrm{O}_{4}$ (gef.: $\mathrm{C} 71,12 \%$, $\mathrm{H} 4,63 \%$; ber. C $71,6 \%, \mathrm{H} 4,47 \%$ ). Nach Säurebehandlung wird sie unverändert zurückerhalten. Mit Essigsäureanhydrid-Pyridin bildet sie ein Monoacetat vom Schmelzpunkt 162 bis $165^{\circ} \mathrm{C}$.

Zum Unterschied von Liquiritigenin zeigt das IRSpektrum der Verbindung bei 1610 und $954 \mathrm{~cm}^{-1}$ Banden, die einer konjugierten $\mathrm{C}=\mathrm{C}-\mathrm{Bindung}$ zuzuordnen sind. Die CO-Bande liegt bei $1620 \mathrm{~cm}^{-1}$.

Das UV-Spektrum besitzt ein starkes Maximum und ein schwaches Nebenmaximum bei 250 bzw. $300 \mathrm{~nm}$, sowie eine Schulter bei $242 \mathrm{~nm}$. Lage und Intensitäten dieser Maxima deuten auf ein Isoflavon hin ${ }^{2}$. Zum Beweis wurde das Absorptionsspektrum des Acetates herangezogen, da die Acetylierung die Beeinflussung des Spektrums durch freie Hydroxylgruppen aufhebt und so das Spektrum des flavonoiden Grundkörpers hervortreten lässt ${ }^{2}$. Das Spektrum zeigt ein Maximum bei $252 \mathrm{~nm}$ und Inflektionen bei 300 und $235 \mathrm{~nm}$. Bei Gegenwart von Natriumacetat wird das Spektrum der acetylierten Sub$\operatorname{stan} z$ nicht verändert. Die nicht acetylierte Verbindung erleidet dagegen eine bathochrome Verschiebung um 10 $\mathrm{nm}$, die ein freies Hydroxyl in 7-Stellung vermuten lässt.

Beim Abbau mit wässriger $\mathrm{KOH}$ und Wasserstoffperoxyd ${ }^{3}$ kann Anissäure papierchromatographisch als Abbauprodukt nachgewiesen werden. Das Seitenphenyl trägt demnach in 4'-Stellung eine Methoxylgruppe. Nach Schmelzpunkt, Spektren und Reaktionen handelt es sich um 7-Hydroxy-4'-methoxy-isoflavon (Formononetin).

Aus der formononetinhaltigen Fraktion des methanolischen Extraktes liessen sich weiterhin etwa $2 \mathrm{mg}$ Ferulasäure isolicren und durch Papierchromatographie und IR-Spektrum charakterisieren.

Den Isoflavonen kommt nach den Untersuchungen von Micheli et al. ${ }^{4}$ eine geringe Östrogenwirksamkeit zu. Die für Süssholz-Extrakte diskutierte östrogene Wirkung 5 konnte aber von HöLLER, Hückel und SCHNEIDER ${ }^{6}$ nicht nachgewiesen werden. Die Aktivität des Formononetins reicht aber nicht aus, um einen solchen Effekt bei Süssholzextrakten manifest werden zu lassen.

Summary. Small amounts of 7-hydroxy-4'-methoxyisoflavone (formononetin) and ferulic acid were isolated from liquorice root and identified by spectral properties and degradation.

W. REINERS

Institut für Pharmazie und Lebensmittelchemie der Universität, Würzburg (Deutschland), 23. Dezember 1965.

1 Als Mitteilung I gilt Naturwiss. 51, 193 (1964).

a L. JuRd, in Spectral Properties of Flavonoidal Componnds (Ed., T. A. Geissman; Pergamon Press, Oxford-London-New YorkParis 1962), p. 107.

3 O. H. Stamm, H. Schmin und J. Büchi, Helv, chim. Acta 47, 2006 (1958).

4 R. A. Micheli, A. N. Booth, A. L. Livingston und E. M. Bickoff, J. med. pharm. Chem. 5, 321 (1962).

5 C. H. Costello und E. V. Lynn, J. Am. pharm. Ass., Sci. Edition $39,177(1950)$

6 H. Höller, H. Hôckel und W. Schneider, Scientia pharm. 28, $33(1960)$. 